\title{
Renal Involvement in Paroxysmal Nocturnal Hemoglobinuria
}

\author{
S. Hussain ${ }^{\mathrm{a}} \quad$ A. Qureshi ${ }^{\mathrm{a}} \quad$ J. Kazi ${ }^{\mathrm{b}}$ \\ ${ }^{a}$ Department of Nephrology, Aga Khan University Hospital Karachi, and b Department of Histopathology, \\ Sindh Institute of Urology and Transplantation, Karachi Medical and Dental College, Karachi, Pakistan
}

\section{Key Words}

Paroxysmal nocturnal hemoglobinuria - Acute kidney injury · Chronic kidney disease $\cdot$ Hemodialysis

\begin{abstract}
Renal involvement in paroxysmal nocturnal hemoglobinuria $(\mathrm{PNH})$ is not usually apparent but in cases with clinical involvement varies from reversible acute dysfunction to chronic irreversible damage. Early diagnosis and treatment is crucial to prevent disease progression and irreversible chronic kidney disease (CKD). The ultimate outcome of CKD in many patients is the need for renal replacement therapy, which necessitates ever-growing dialysis and transplantation programs, thereby imposing a significant economic burden on the healthcare system. In a third-world country like Pakistan, increased burden due to CKD can be very hard on families. Doctor visits, hospitalization, and dialysis are all out-of-pocket expenses, therefore prevention, early detection, and timely intervention are the only cost-effective strategies. We report a case of acute kidney injury (AKI) due to $\mathrm{PNH}$. This case shows AKI as one of the complications of PNH which may have a clinical course like acute tubular necrosis (ATN). This could be due to ATN or AKI superimposed on CKD due to hemosiderin deposits in the renal tubular epithelial cells. Our patient was dialyzed initially and discharged with a permanent catheter in place with advice to continue dialysis three times a week. He required dialysis for 1 week then started producing urine. His subsequent outpatient visits showed improved renal function. The permanent catheter was removed and maintenance dialysis was stopped. Here,
\end{abstract}

we briefly review the literature on renal involvement in $\mathrm{PNH}$, treatment options for $\mathrm{PNH}$ and pigment-induced nephropathy followed by a question-and-answer session at the clinicopathological conference held on March 4, 2011, at Sindh Institute of Urology and Transplantation in Karachi, Pakistan.

Copyright $\odot 2013$ S. Karger AG, Basel

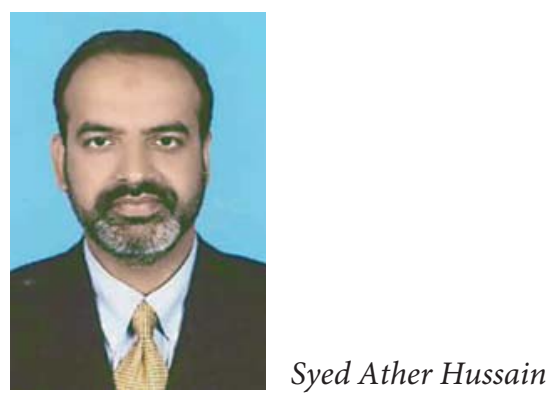

\section{Biography}

Syed Ather Hussain, 41 years old, graduated (1994) in Medicine from Dow Medical College Karachi, Pakistan. He went on to complete his Residency in Internal Medicine (1996-2000) and Nephrology (2000-2003) at the Medical College of Wisconsin, USA. He then did Masters in Science from the Department of Epidemiology at the Medical College of Wisconsin. He is currently working as a consultant nephrologist at the Aga Khan University Hospital as an Associate Professor of Medicine and is Section Head of the Nephrology Section, Department of Medicine.

\section{KARGER}

E-Mail karger@karger.com

www.karger.com/nec
(C) 2013 S. Karger AG, Basel

$1660-2110 / 13 / 1232-0028 \$ 38.00 / 0$
Dr. Abdul Rehman Qureshi

Aga Khan University Hospital Karachi

POB 3500, Stadium Road

Karachi 74800 (Pakistan)

E-Mail rehman.qureshi@aku.edu 


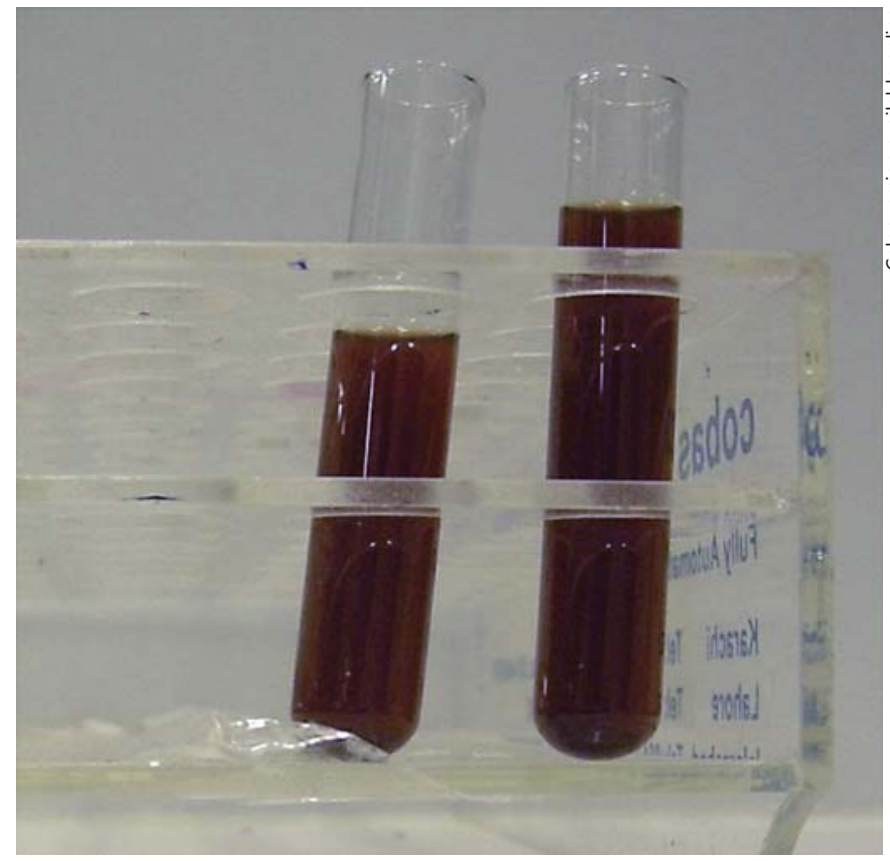

Fig. 1. Cola colored urine.

Table 1. Investigations at the time of presentation

\begin{tabular}{ll}
\hline BUN, mmol/l & 70.6 \\
Serum creatinine, $\mu \mathrm{mol} / \mathrm{l}$ & $2,015.5$ \\
Serum sodium, mmol/l & 125 \\
Serum potassium, $\mathrm{mmol} / \mathrm{l}$ & 5.8 \\
Serum chloride, $\mathrm{mmol} / \mathrm{l}$ & 91 \\
Serum bicarbonate, $\mathrm{mmol} / \mathrm{l}$ & 12.6 \\
CBC & \\
$\quad$ Hemoglobin, g/l & 56 \\
$\quad$ Hematocrit, $\%$ & 16.7 \\
$\quad$ Mean cell volume, fl & 86 \\
$\quad$ WBC, l & $4.2 \times 10^{9}$ \\
$\quad$ Platelet count, 1 & $250 \times 10^{9}$ \\
Serum albumin, g/l & 33 \\
Serum calcium, mmol/l & 1.7 \\
Serum phosphate, $\mu \mathrm{mol} / \mathrm{l}$ & 3.64 \\
Random blood sugar, $\mathrm{mmol} / \mathrm{l}$ & 4.66 \\
\hline
\end{tabular}

\section{Case Presentation}

\section{Dr. A. Qureshi}

A 48-year-old Pakistani male with a history of paroxysmal nocturnal hemoglobinuria $(\mathrm{PNH})$ was electively admitted for blood transfusion on January 13, 2011, at Aga Khan University Hospital Karachi. He had a history of dark cola-colored urine (fig. 1), high-grade fever, and a productive cough for 3 days. These symptoms were associated with generalized abdominal pain, nausea, vomiting, and shortness of breath at mild exertion. His past history included PNH since 2000 diagnosed by absent CD55 and CD59 assay by flow cytometry of red blood cells (RBCs). He required blood transfusion at an average of 6-month intervals. Approximately 20-25 packed RBCs had been transfused since the diagnosis of PNH. During this admission he was transfused with 3 packed RBCs.

On physical examination at admission he was afebrile with a temperature of $37^{\circ} \mathrm{C}$ (as he had been given antipyretics), had a heart rate of $75 \mathrm{bpm}$, respiratory rate of $20 \mathrm{breaths} / \mathrm{min}$, blood pressure of $124 / 68 \mathrm{~mm} \mathrm{Hg}$, and oxygen saturation of $99 \%$ in room air. He was pale, non-edematous with tenderness in the left lumber region and the left kidney was also palpable. The rest of the physical examination was unremarkable.

On the basis of his history and clinical examination, the following differential diagnoses were established. As the presenting symptom was cola-colored urine, pigment-induced nephropathy was therefore the first differential, followed by glomerulonephritis (e.g. IgA nephropathy or postinfectious glomerulonephritis) and pulmonary-renal syndrome (granulomatosis with polyangitis, microscopic polyangitis, Goodpasture's syndrome) due to simultaneous involvement of lungs. There was the possibility of hypotension-induced acute tubular necrosis (ATN) but there was no documented episode of hypotension. There was no history of fever, jaundice, petechial rash, hepatomegaly or bleeding, therefore clinical diagnoses of malaria, dengue fever, hepatitis or platelets disorder were less likely. There was also no history of trauma/excessive exercise which could lead to myoglobinuria. Although the left kidney was palpable the patient had no history of hypertension or family history of intracranial hemorrhage or adult polycystic kidney disease. As only the solitary kidney was palpable, there were possibilities of nephrolithiasis leading to obstruction, pyelonephritis, renal vein thrombosis, or renal cell carcinoma. Some other possible causes of enlarged kidneys were HIV nephropathy, multiple myeloma, lymphoma and diabetes. These differentials were kept in mind prior to ordering the workup.

His laboratory data at admission are outlined in table 1. A previous renal function test was done in 2007 which showed 97.24 $\mu \mathrm{mol} / \mathrm{l}$ serum creatinine and $4.99 \mathrm{mmol} / \mathrm{l} \mathrm{BUN}$. Urinalysis showed a dark brown color appearance, $\mathrm{pH}$ 5.5, specific gravity 1.015, 10 RBCs and 8 white blood cells (WBCs) per high-power field, without any cast, urinary ketone and glucose were negative, urine protein $1.0 \mathrm{~g} / \mathrm{l}$, and the spot urinary protein and creatinine ratio was $2 \mathrm{~g} / \mathrm{g}$ creatinine. The sample was catheterized and concentrated, and the patient was oliguric (urine output was $300 \mathrm{ml}$ in the last $24 \mathrm{~h}$ ) at the time of presentation, so the presence of RBCs could be either due to a trauma or associated with glomerulonephritis. Hemosiderin stain in urine was also positive (fig. 2). Further laboratory findings showed lactate dehydrogenase $130.4 \mu \mathrm{kat} / \mathrm{l}$ with a reticulocyte count of $5.1 \%$, uric acid 3,537.8 $\mu \mathrm{mol} / \mathrm{l}$, total bilirubin $17.1 \mu \mathrm{mol} / \mathrm{l}$ and Coomb's test was negative. His blood and urine cultures were negative; serum tested negative for hepatitis B surface antigen and hepatitis $C$ virus antibodies, and his ANA profile done in 2007 was negative.

An ultrasound scan revealed bilateral enlarged kidneys with poor corticomedullary differentiation. The right kidney was 14.8 $\mathrm{cm}$ in size and the left kidney was $16.5 \mathrm{~cm}$. There was poor vascularity on ultrasound Doppler but no specific renal vein Doppler was done to rule out renal vein thrombosis. There was no evidence 


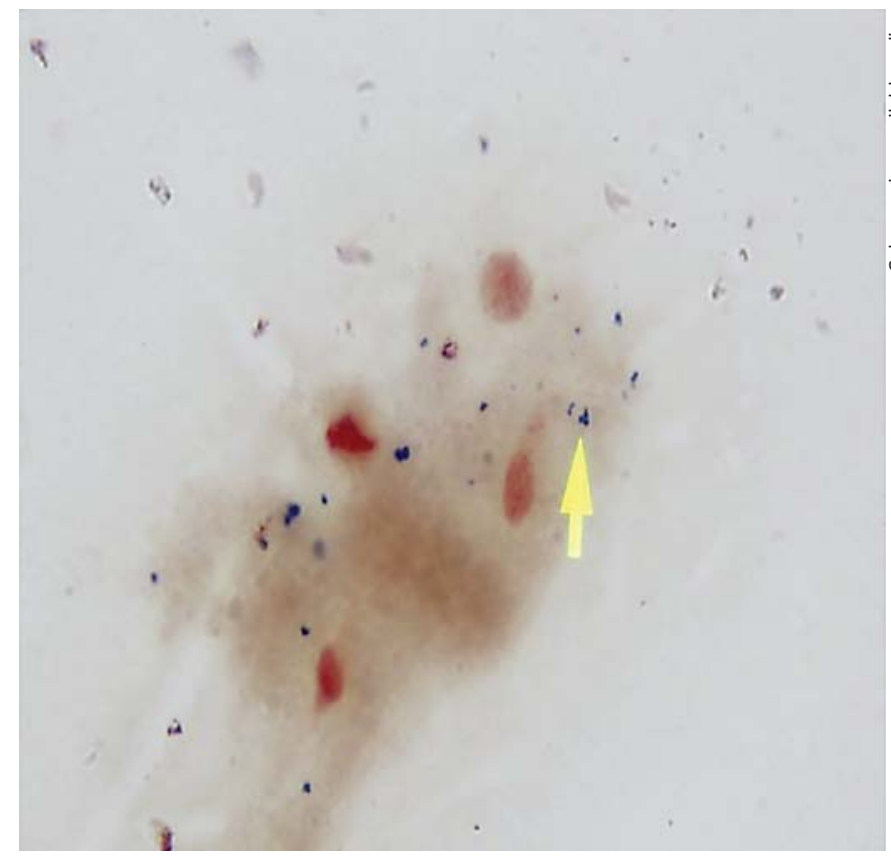

Fig. 2. Arrow showing hemosiderin stain in urine.

of mass, cyst, obstruction or stone on renal ultrasound. The liver was normal in size and texture. Chest $\mathrm{x}$-ray was also normal except for hilar congestion with no evidence of alveolar infiltrates.

A renal biopsy was performed to further elucidate the reason for renal failure (fig. 3-5), but electron microscopy could not be performed as such a facility is not available at Aga Khan University Hospital Karachi.

\section{Dr. J. Kazi}

A renal biopsy specimen was an adequate sample and included the cortex and medulla with twelve glomeruli in a serial section, ten of which were globally sclerosed. The remaining two glomeruli appeared normocellular with normal capillary wall thickening. There was moderate patchy tubular atrophy associated with interstitial scarring and inflammation. In addition, there were many nephrons which were hypertrophic and showed features of acute tubular injury and gold-brown pigment deposition in renal tubular epithelial cells. This was iron as demonstrated by positive reaction on prussian blue staining. There were no ischemic changes in the glomeruli blood vessels. There was no staining of immunoglobulins and complement on immunofluorescence. The morphological findings in the biopsy most probably suggest chronic sclerosing glomerulonephritis as the underlying disorder with superimposed acute tubular injury caused by excess iron deposition in the hypertrophic nephrons. It is not possible to determine the relationship of the acute and chronic changes from the morphological study of the renal biopsy only.

\section{Dr. A. Qureshi}

Initially the patient was hydrated with normal saline (2-3 liters of saline were given over $12 \mathrm{~h}$ ) and hyperkalemia was managed

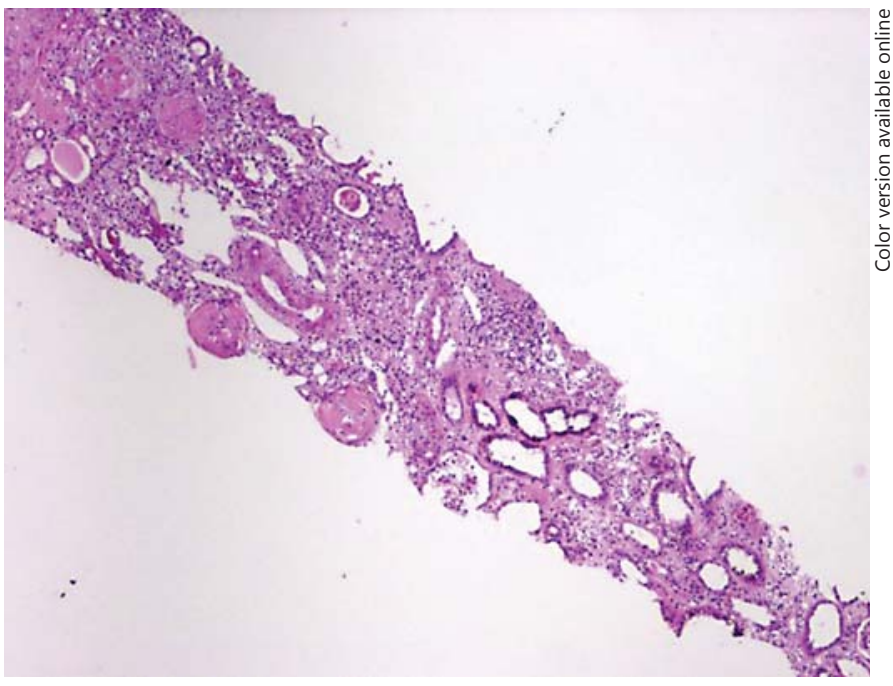

Fig. 3. Core of renal biopsy showing sclerosed glomeruli.

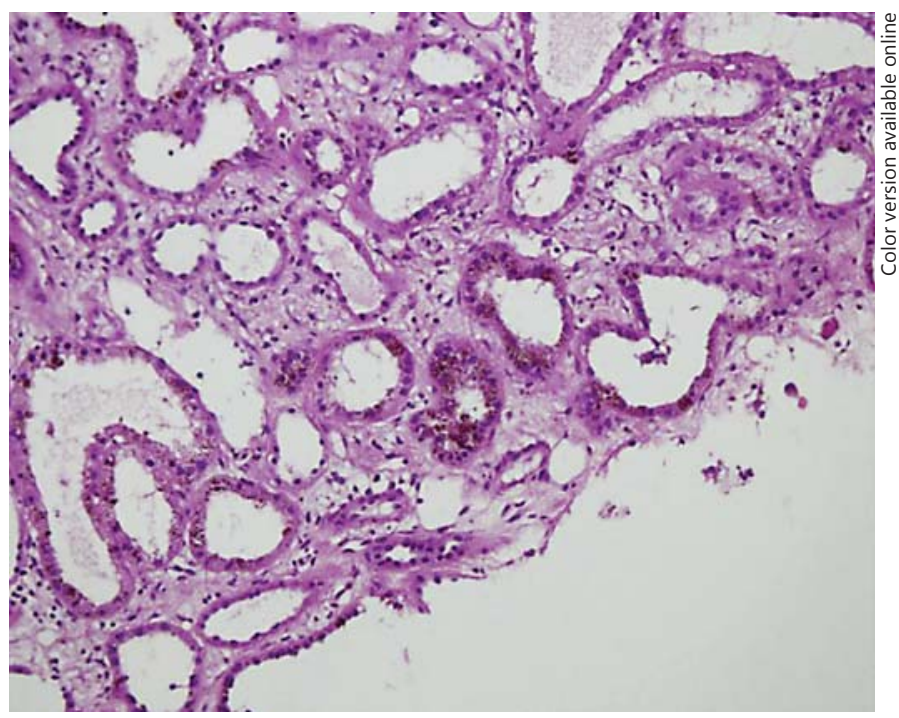

Fig. 4. Tubular atrophy and interstitial inflammation. 


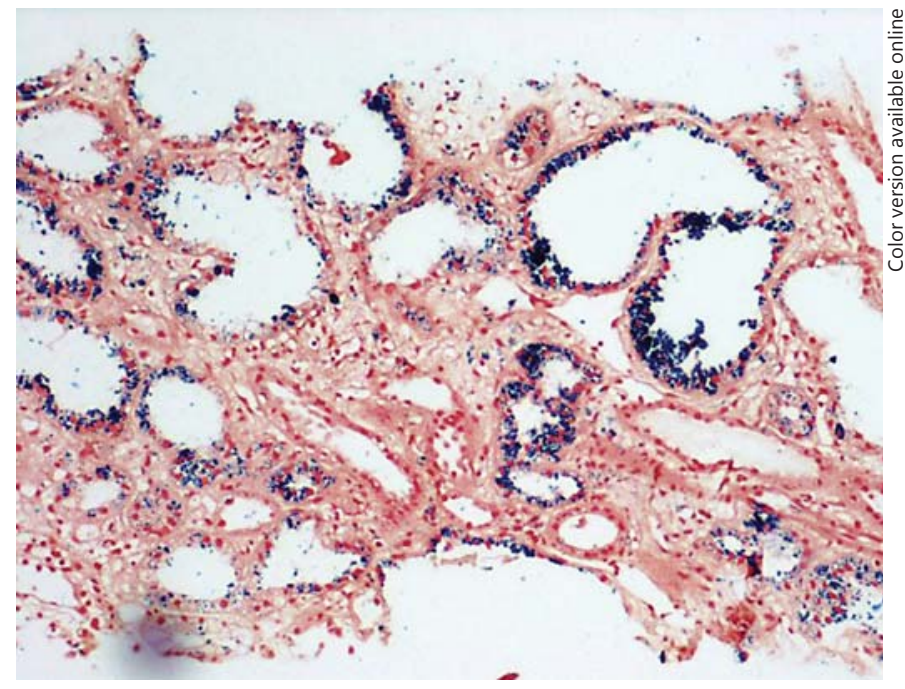

Fig. 5. Pigment deposition in renal tubules.

Table 2. Outpatient investigations

\begin{tabular}{ll}
\hline BUN, mmol/l & 16.77 \\
Serum creatinine, $\mu \mathrm{mol} / \mathrm{l}$ & 282.8 \\
Serum sodium, $\mathrm{mmol} / \mathrm{l}$ & 137 \\
Serum potassium, $\mathrm{mmol} / \mathrm{l}$ & 4.0 \\
Serum chloride, $\mathrm{mmol} / \mathrm{l}$ & 110 \\
Serum bicarbonate, $\mathrm{mmol} / \mathrm{l}$ & 21 \\
\hline
\end{tabular}

medically. Hemodialysis was initiated once he remained oliguric with deranged renal functions despite of conservative management. He was dialyzed initially (a total of 5 sessions as an inpatient) and discharged with a permanent tunneled catheter placed in the right jugular vein with the advice to continue dialysis three times a week. He required dialysis for 1 more week (a total of 3 sessions) and then started producing urine. His subsequent outpatient visit showed improved renal function (table 2). The permanent catheter was removed and maintenance dialysis was stopped. The patient's last serum creatinine level was $1.1 \mathrm{mg} / \mathrm{dl}$ which reflects an improved renal function (fig. 6).

\section{Case Discussion}

\section{S. Hussain}

Acute kidney injury (AKI) can be induced by the release of heme pigments, as with myoglobinuria due to rhabdomyolysis and hemoglobinuria due to intravascular hemolysis [1]. The incidence of AKI related to hemolysis is not well described, but may be as high as $50 \%$ with massive hemolysis $[2,3]$. PNH is one of the causes of intravascular hemolysis.

$\mathrm{PNH}$ is a rare disorder with a minimum prevalence estimated to affect $1-1.5$ cases per million population. The diagnosis is often made in East Asian countries, but it is not certain whether the incidence is greater in this region [4]. PNH has the same general epidemiology as aplastic anemia [4], i.e. both are most common in young adults but later have a secondary increase in the seventh decade [5]. $\mathrm{PNH}$ has a male preponderance and the incidence of arterial and venous thrombosis is relatively rare [6].

$\mathrm{PNH}$ is a disorder characterized by a defect in the glycosylphosphatidylinositol (GPI) anchor due to an abnormality in the Pig-a gene. This leads to partial or complete absence of certain GPI-linked proteins, particularly CD59 (also called membrane inhibitor of reactive lysis, protectin - a complement regulatory protein, and membrane attack complex inhibitory factor) and CD55 (decay accelerating factor) [7]. The clinical syndrome can present in three types of symptoms including (1) an acquired intracorpuscular hemolytic anemia due to the abnormal susceptibility of the red cell membrane to the hemolytic activity of complement, (2) thromboses in large vessels, such as hepatic, abdominal, cerebral, and subdermal veins, and (3) a deficiency in hematopoiesis that may be mild or severe, such as pancytopenia in the aplastic anemia state. The triad of hemolytic anemia, pancytopenia, and thrombosis makes PNH a truly unique clinical syndrome. 
Intravascular hemolysis in $\mathrm{PNH}$ can lead to two forms of renal disease: (1) a severe hemolytic episode of any cause (often in association with gastroenteritis) with massive hemoglobinuria can cause AKI probably from ATN $[8,9]$, and (2) chronic hemolysis results in iron deposition in the kidneys in almost all patients. Iron deposition can result in proximal tubular dysfunction [8-10]. In addition, chronic renal failure due to hemosiderosis and subsequent interstitial scarring can occur in patients with long-standing PNH [10-14]. Electron microscopy of renal parenchyma confirms the loss of proximal tubular brush borders and demonstrates decreased infoldings at the basolateral membrane of proximal tubular epithelial cells.

As indicated in the above-mentioned literature, our patient presented with markedly deranged renal functions with very high creatinine, enlarged kidney possibly due to congestion, hemosiderin in urine and its deposition in renal tubular epithelium with marked glomerulosclerosis which was an unusual presentation. There is evidence reporting that patients with $\mathrm{PNH}$ and less advanced stages of AKI can be managed successfully without hemodialysis [15]. There is a case report of a 57-year-old male who presented with diarrhea, darkened urine, jaundice and increased blood urea nitrogen and creatinine, whose renal biopsy indicated ATN and hemosiderin deposition. A CD55 and CD59 assay, Ham test and sugarwater hemolysis test confirmed the diagnosis of $\mathrm{PNH}$. He was managed conservatively with fluids, diuresis and urine alkalization and gradually regained near-normal renal function [15], but was oliguric with advanced AKI and required urgent hemodialysis. Moreover, the renal pathology sample showed the most affected area of the kidney (10 out of 12 glomeruli sclerosed) which misrepresented the whole kidney histology as his AKI resolved showing that he probably had much more viable renal tissue.

There are case reports that show occurrence of AKI and chronic kidney disease (CKD) due to PNH. In all these cases a diagnosis of PNH was made after detection of renal dysfunction [16]. There is a case report from India in which a 70-year-old male had had chronic idiopathic thrombocytopenic purpura since 1990 and who presented with low hemoglobin $(\mathrm{Hb})$. He was found to have PNH and AKI. The patient was adequately hydrated and put on prednisone at a dose of $1 \mathrm{mg} / \mathrm{kg}$. Over the next 3 weeks, renal functions normalized spontaneously without any further intervention. Hb gradually rose to $8 \mathrm{~g} / \mathrm{dl}$ without any transfusions. The patient continued a regular follow-up and maintained normal renal parameters [16].
In another case, a 49-year-old male from India with the diagnosis of aplastic anemia was found to have had darkcolored urine for 10 months. Investigations revealed $\mathrm{PNH}$ with AKI. He was treated with packed RBC transfusion and also received two sessions of hemodialysis. He continued to have normal renal parameters 10 weeks after the illness [16]. Another case, a 51-year-old hypertensive male with CKD, presented with dyspnea and was found to have $\mathrm{PNH}$. He did not require hemodialysis and was adequately hydrated and started on prednisone $(1 \mathrm{mg} /$ $\mathrm{kg}$ ), danazol and erythropoietin. His anemia improved and after 3 months of treatment and follow-up, serum creatinine stabilized at $176.8 \mu \mathrm{mol} / 1$ [16]. CKD in our patient could not be established due to the large size and absence of recent baseline renal functions. Our patient was lost to follow-up after 2 months of presentation and his last available creatinine was $97.24 \mu \mathrm{mol} / \mathrm{l}$ with an estimated glomerular filtration rate (calculated by Cockcroft-Gault equation [17]) of $76.5 \mathrm{ml} / \mathrm{min}$, so CKD criteria over the timeframe could not be established. His high phosphate and low calcium levels could be due to intravascular hemolysis, deposition of calcium in tissues and calcium phosphate product formation. His low $\mathrm{Hb}$ is due to $\mathrm{PNH}$ and he had been transfused blood products, so an association of low $\mathrm{Hb}$ and the relationship with $\mathrm{CKD}$ would be difficult. Once the patient is more stable, repetition of protein estimation is warranted since he had protein $2 \mathrm{~g} / \mathrm{g}$ creatinine in the spot urine sample, in the presence of oliguria and 20-30 RBCs.

There have been cases in which PNH is associated with glomerular diseases, such as IgA nephropathy, focal segmental glomerular sclerosis and membranous nephropathy [18-20]. In this case, renal biopsy did not show focal and segmental proliferation of mesangial cells, mesangial matrix expansion, focal and segmental sclerosis or membranous glomerulopathy, and there was no staining of immunoglobulins and complement on immunofluorescence.

The general goals for preventive therapy in heme pigment-induced acute renal failure are correcting volume depletion and preventing intratubular cast formation. Early recognition of AKI and its immediate management with fluid resuscitation is important as persistent kidney injury predisposes to long-term consequences [21-24]. $\mathrm{AKI}$ is a complex disorder that occurs in a variety of settings with clinical manifestations ranging from a minimal elevation in serum creatinine to anuric renal failure. It is often underrecognized and is associated with severe consequences [21-24]. It is associated with the increased mortality, particularly when dialysis is required [21-24]. 
The proposed diagnostic criteria of AKI is an abrupt (within $48 \mathrm{~h}$ ) reduction in kidney function currently defined as an absolute increase in serum creatinine $\geq 0.3$ $\mathrm{mg} / \mathrm{dl}(\geq 26.4 \mu \mathrm{mol} / \mathrm{l})$, a percentage increase in serum creatinine of $\geq 50 \%$ ( 1.5 -fold from baseline), or a reduction in urine output (documented oliguria $<0.5 \mathrm{ml} / \mathrm{kg} \cdot \mathrm{h}$ for $>6$ h) [25]. Recently, a consensus conference sponsored by the Acute Dialysis Quality Improvement Initiative (ADQI) has proposed a new definition of AKI, which has been widely endorsed and is increasingly being used [26]. In keeping with the spectrum of changes seen in AKI, a diagnostic classification scheme was developed. This scheme is referred to by the acronym RIFLE, and includes three levels of renal dysfunction of increasing severity, namely Risk of renal dysfunction, Injury to the kidney and Failure of kidney function, and two outcome categories: Loss of function and End-stage kidney disease. There has been a marked difference in etiologies of AKI in different parts of the world. Surgery and trauma are the most common causes of AKI in developed countries [27-29]. Recently, the incidence of surgical and posttraumatic AKI has decreased, whereas that of medical AKI has increased. In developing countries like Pakistan, medical causes account for $88 \%$ of AKI and surgical causes for $11 \%$ [30]. Of the medical causes, sepsis (25\%) and dehydration secondary to diarrhea and vomiting (21\%) are the most common etiological factors of AKI which results in hemodialysis in $17 \%$ patients in each category.

There are two modalities that have been used to prevent or minimize the severity of AKI: volume repletion and a forced alkaline-mannitol diuresis [31]. Adequate fluid resuscitation is very important for preventing AKI. The goals of volume repletion are to both enhance renal perfusion (thereby minimizing ischemic injury) and increase the urine flow rate to wash out obstructing casts [31]. A urine output of $200-300 \mathrm{ml} / \mathrm{h}$ is desirable while myoglobinuria (discolored urine) persists [31]. Experimental studies suggest that mannitol might be protective primarily by causing a diuresis, which minimizes intratubular heme pigment deposition and cast formation [32]. Studies show no amelioration of proximal tubular necrosis with mannitol, but may cause hyperosmolality and other complications [32]. Our patient was not administered mannitol as he was oliguric and was initially hydrated with saline. All possible methods were used to prevent AKI, including maintenance of fluid volume and renal perfusion pressure, as even mild forms of AKI are associated with high mortality rates [25]. Hemodialysis was initiated when he remained oliguric with deranged renal functions after conservative management.

Renal Involvement in Paroxysmal

Nocturnal Hemoglobinuria
As mentioned, CKD criteria over the timeframe were not established as the patient was unfortunately lost to follow-up. CKD has become a major public health problem worldwide $[33,34]$. The social and economic implications of CKD are substantial not only due to morbidity and mortality associated with the progression to kidney failure, but also because of its association with accelerated cardiovascular disease [35]. The ultimate outcome of $\mathrm{CKD}$ in many patients is the need for renal replacement therapy which necessitates ever-growing dialysis and transplantation programs, thereby imposing a significant economic burden on the healthcare system. In a thirdworld country like Pakistan, with a total GDP USD 211.1 billion [36] and only $1.76 \%$ is reserved for healthcare [36], an increased burden due to CKD can be very hard on families. Doctor visits, hospitalization and dialysis are all out-of-pocket expenses. Overall, per-capita income is about USD 672 [36] and the annual cost of dialysis of a single patient is over USD 3,000 [37]. Prevention, early detection, and timely intervention are therefore the only cost-effective strategies. AKI appears to be associated with a higher risk of CKD even among relatively low-risk patients. This was suggested by a cohort study of 1,610 patients who developed in-hospital AKI [38]. Patients selected for the analysis had normal renal function prior to AKI and recovered to within $90 \%$ of pre-AKI estimated glomerular filtration rate within 90 days; $81 \%$ of the patients recovered baseline renal function within 4 days. Patients who developed AKI were more likely to develop CKD by 3.3 years compared with matched control patients who did not develop AKI (hazard ratio 1.9). The same result was observed in a larger cohort study of 3,809 patients with normal baseline renal function, in which AKI was associated with a higher risk of CKD at 2.5 years (hazard ratio by propensity score-stratified analysis, 3.82, 95\% CI 2.81-5.19) [39]. It cannot be determined from either of these observational studies whether AKI caused CKD or whether the association of AKI with subsequent CKD is due to common risk factors [40]. The previously established perception that patients, particularly those with previous normal function, who recover from AKI return to or near to their baseline kidney function has been increasingly called into question. Multiple studies have demonstrated an increase in the risk of CKD and end-stage renal disease (ESRD) among patients who recover from AKI.

To our knowledge, there is limited data with renal replacement therapy in patients with ESRD due to PNH. Vanwalleghem et al. [41] have discussed the case of renal transplantation in a 60-year-old patient with $\mathrm{PNH}$ in

Nephron Clin Pract 2013;123:28-35 DOI: $10.1159 / 000351345$ 
whom there was significant reduction in hemolytic activity in the posttransplant period, and the patient became transfusion-independent. However, during a 9-month follow-up, there were three major hemolytic crises and evidence of renal cortical hemosiderosis in the MRI scans.

The only potentially curative treatment for $\mathrm{PNH}$ is allogeneic hematopoietic cell transplantation. All other treatments are supportive and are directed against the major manifestations of the disease [31]. Because hemoglobinuria is a prominent complication of $\mathrm{PNH}$, all patients with PNH should be evaluated for the presence of iron deficiency [31], and if found, should be treated with iron supplementation [31]. We have not come across any recommendations for the role of chelating agents (such as deferoxamine, deferasirox and deferiprone) in patients with $\mathrm{PNH}$ requiring multiple blood transfusions except when the ferritin level exceeds $1,000 \mathrm{ng} / \mathrm{ml}$ as in $\beta$ thalassemia [31]. As a general precaution, oral folic acid supplementation ( $1 \mathrm{mg} /$ day) should be given prophylactically to those with hemolytic anemia in order to avoid the development of folic acid deficiency [31]. For patients with symptomatic anemia due to $\mathrm{PNH}$, supportive treatment with periodic blood transfusions or the use of erythropoiesis-stimulating agents, androgens, and/or glucocorticoids have all been helpful in alleviating the signs and symptoms of anemia [31]. There are no randomized studies that give guidance on the selection of an appropriate agent for this complication of PNH. For selected transfusion-dependent patients or patients with disabling symptoms (e.g. fatigue, thromboses, frequent paroxysms of pain, end-organ damage), treatment with eculizumab is suggested [42]. Our patient was discharged on iron supplement, folic acid, and oral prednisone at $30 \mathrm{mg} /$ day.

\section{Conclusions}

Dr. S. Hussain: This case shows that PNH may present with AKI when hemolysis occurs and hemosiderin deposits in the renal tubular epithelial cells. Early diagnosis and treatment are crucial to prevent disease progression and irreversible CKD.

\section{Questions and Answers}

Question 1: Question posed by Dr. Dilip Kumar from Jinnah Postgraduate Medical Center (JPMC), Karachi, Pakistan:

Is this patient a suitable candidate for renal transplant?
Answer by Dr. S. Hussain: As we mentioned, the patient was diagnosed with PNH 10 years earlier and presented to us with AKI with partial recovery of renal function. This shows that kidney damage may occur at any point of time in patients with PNH. Data shows that patient survival is much longer after renal transplant compared to if kept on dialysis [43]. Patients with PNH and ESRD are therefore suitable candidates for renal transplant.

Question 2: Question posed by Dr. Aftab Khan from Sheikh Zayed Hospital, Lahore, Pakistan:

Would you repeat renal biopsy in this patient to evaluate the extent of disease as the patient recovered but his renal biopsy specimen showed marked sclerosis and damage?

Answer by Dr. S. Hussain: Yes, renal biopsy can be repeated to histologically confirm the reversibility of disease and presence of viable renal parenchyma, but this would be only for academic purposes. Our patient clinically improved, with improved serum creatinine and was taken off dialysis. We suspect clinical reversibility of the disease process.

Question 3: Question posed by Dr. Sonia Yaqub from Aga Khan University Hospital, Karachi, Pakistan:

Would you repeat the renal ultrasound and why?

Answer by Dr. S. Hussain: Renal ultrasound in this patient can be repeated to evaluate the reduction in renal size, especially with improvement in renal function which may reflect a reduction in the inflammatory process and edema.

Question 4: Question posed by Dr. Tanveer Khan from Sindh Institute of Urology and Transplantation, Karachi, Pakistan:

As you mentioned that there was hemosiderin deposition in renal tubules and he was discharged on iron supplements, we want to know if he should be given iron chelating agents which may prevent iron deposition in other organs?

Answer by Dr. S. Hussain: In PNH there is continuous loss of $\mathrm{Hb}$ in urine with iron loss and the patient may have iron deficiency anemia. Our patient's iron studies showed $870 \mu \mathrm{g} / \mathrm{l}$ serum ferritin and $301 \mu \mathrm{mol} / \mathrm{l} \mathrm{TIBC}$, and these samples were sent after blood transfusions. Yes, the patient had hemosiderin deposition in renal tubules but his liver ultrasound was normal and there were no stigmata of iron deposition elsewhere, for example his skin was not tanned, blood sugars were normal, and there was no cardiomegaly or joint involvement. As mentioned earlier, we have not come across any recommendations for the role of chelating agents in patients with $\mathrm{PNH}$ requiring multiple blood transfusions except when the ferritin level exceeds $1,000 \mathrm{ng} / \mathrm{ml}$ as in $\beta$-thalassemia [31].
Hussain/Qureshi/Kazi 


\section{References}

1 Zager RA: Studies of mechanisms and protective maneuvers in myoglobinuric acute renal injury. Lab Invest 1989;60:619-629.

$>2$ Centers for Disease Control and Prevention: Hemolysis associated with $25 \%$ human albumin diluted with sterile water - United States, 1994-1998. JAMA 1999;281:1076-1077.

$\checkmark 3$ Choudhry VP, Ghafary A, Zaher M, et al: Drug-induced haemolysis and renal failure in children with glucose-6-phosphate dehydrogenase deficiency in Afghanistan. Ann Trop Paediatr 1990;10:335-338.

4 Issaragrisil S, Leaverton PE, Chansung K, et al: Regional pattern in the incidence of aplastic anemia in Thailand. Am J Hematol 1999; 61:164-168.

$\checkmark 5$ Nishimura J, Kanakura Y, Ware RE, et al: Clinical course and flow cytometric analysis of paroxysmal nocturnal hemoglobinuria in United States and Japan. Medicine 2004;83: 193-207.

6 Kruatrachue M, Wasi P, Na-Nakorn S: Paroxysmal nocturnal haemoglobinuria in Thailand with special reference to an association with aplastic anaemia. Br J Haematol 1978;39: 267-276.

7 Rosse WF: Paroxysmal nocturnal hemoglobinuria as molecular disease. Medicine (Baltimore) 1997;76:63-93.

$>8$ Jackson GH, Noble RS, Maung ZT, et al: Severe haemolysis and renal failure in a patient with paroxysmal nocturnal haemoglobinuria. J Clin Pathol 1992;45:176-177.

$\checkmark 9$ Mooraki A, Boroumand B, Mohammad Zadeh F, et al: Acute reversible renal failure in a patient with paroxysmal nocturnal hemoglobinuria. Clin Nephrol 1998;50:255-257.

10 Clark DA, Butler SA, Braren V, et al. The kidneys in paroxysmal nocturnal hemoglobinuria. Blood 1981;57:83-89.

11 Riley AL, Ryan LM, Roth DA: Renal proximal tubular dysfunction and paroxysmal nocturnal hemoglobinuria. Am J Med 1977;62:125129.

-12 Hsiao PJ, Wang SC, Wen MC, et al: Fanconi syndrome and $\mathrm{CKD}$ in a patient with paroxysmal nocturnal hemoglobinuria and hemosiderosis. Am J Kidney Dis 2010;55:e1.

13 Zachée P, Henckens M, Van Damme B, et al: Chronic renal failure due to renal hemosiderosis in a patient with paroxysmal nocturnal hemoglobinuria. Clin Nephrol 1993;39:2831.

$\checkmark 14$ Hillmen P, Elebute M, Kelly R, et al: Longterm effect of the complement inhibitor eculizumab on kidney function in patients with paroxysmal nocturnal hemoglobinuria. Am J Hematol 2010;85:553-559.

15 Qi K, Zhang XG, Liu SW, et al: Reversible acute kidney injury caused by paroxysmal nocturnal hemoglobinuria. Am J Med Sci 2011;341:68-70.
16 Nair RK, Khaira A, Sharma A, et al: Spectrum of renal involvement in paroxysmal nocturnal hemoglobinuria: report of three cases and a brief review of the literature. Int Urol Nephrol 2008;40:471-475.

17 Levey AS, Bosch JP, Lewis JB, et al: A more accurate method to estimate glomerular filtration rate from serum creatinine: a new prediction equation. Modification of Diet in Renal Disease Study Group. Ann Intern Med 1999;130:461-470.

18 Kato K, Shibata T, Mukai K, et al: Case of paroxysmal nocturnal hemoglobinuria complicated with IgA nephropathy who developed acute renal failure induced by hemolytic crisis. Nihon Jinzo Gakkai Shi 2005;47:540-546.

19 Takahashi K, Yoshimura A, Inoue Y, et al: A case of paroxysmal nocturnal hemoglobinuria combined with focal segmental glomerular sclerosis. Nihon Jinzo Gakkai Shi 2001;43: 39-43.

20 Lee GW, Lee JH, Kim SB, et al: Membranous glomerulopathy as a manifestation of chronic graft-versus-host-disease after non-myeloablative stem cell transplantation in a patient with paroxysmal nocturnal hemoglobinuria. J Korean Med Sci 2003;18:901-904.

21 Mehta RL, Pascual MT, Soroko S, et al: Spectrum of acute renal failure in the intensive care unit: the PICARD experience. Kidney Int 2004;66:1613-1621.

22 Palevsky PM: Epidemiology of acute renal failure: the tip of the iceberg. Clin J Am Soc Nephrol 2006;1:6-7.

23 Ympa YP, Sakr Y, Reinhart K, Vincent JL: Has mortality from acute renal failure decreased? A systematic review of the literature. Am J Med 2005;118:827-832.

24 Metnitz PG, Krenn CG, Steltzer H, et al: Effect of acute renal failure requiring renal replacement therapy on outcome in critically ill patients. Crit Care Med 2002;30:2051-2058.

25 Mehta RL, Kellum JA, Shah SV, et al: Acute Kidney Injury Network: report of an initiative to improve outcomes in acute kidney injury. Crit Care 2007;11:R31.

26 Bellomo R, Ronco C, Kellum J, et al: Acute renal failure - definition, outcome measures, animal models, fluid therapy and information technology needs: the Second International Consensus Conference of the Acute Dialysis Quality Initiative (ADQI) Group. Critical Care 2004;8:R204-R212.

27 Abreo K, Moorthy AV, Osborne M: Changing pattern and outcome of acute renal failure requiring hemodialysis. Arch Intern Med 1986; 146:1338-1344.

28 Turney JH, Marshall DH, Brownjohn AM, et al: The evolution of acute renal failure, 19561988. Q J Med 1990;74:83-104.
29 Beaman M, Turney JH, Rodger RSC, et al: Changing pattern of acute renal failure Q J Med 1987;62:15-23.

30 Rabbani MA, Habib HB, Siddiqui BK, et al: Etiology of acute renal failure in a tertiary center. Saudi J Kidney Dis Transpl 2008;19:10091014.

31 UpToDate, Waltham/MA: www.uptodate. com.

-32 Zager RA: Combined mannitol and deferoxamine therapy for myohemoglobinuric renal injury and oxidant tubular stress. Mechanistic and therapeutic implications. J Clin Invest 1992;90:711-719.

33 Coresh J, Selvin E, Stevens LA, et al: Prevalence of chronic kidney disease in the United States. JAMA 2007;298:2038-2047.

34 Snyder JJ, Foley RN, Collins AJ: Prevalence of CKD in the United States: a sensitivity analysis using the National Health and Nutrition Examination Survey (NHANES) 1999-2004. Am J Kidney Dis 2009;53:218-228.

35 Sarnak MJ, Levey AS, Schoolwerth AC, et al: Kidney disease as a risk factor for development of cardiovascular disease: a statement from the American Heart Association Councils on Kidney in Cardiovascular Disease, High Blood Pressure Research, Clinical Cardiology, and Epidemiology and Prevention. Circulation 2003;108:2154-2169.

36 Trading Economics, New York: www. tradingeconomics.com.

-37 Ali Jaffar Naqvi S: Nephrology services in Pakistan. Nephrol Dial Transplant 2000;15: 769-771.

38 Bucaloiu ID, Kirchner HL, Norfolk ER, et al: Increased risk of death and de novo chronic kidney disease following reversible acute kidney injury. Kidney Int 2012;81:477-485.

39 Jones J, Holmen J, De Graauw J, et al: Association of complete recovery from acute kidney injury with incident CKD stage 3 and allcause mortality. Am J Kidney Dis 2012;60: 402-408.

40 Palevsky PM: Chronic-on-acute kidney injury. Kidney Int 2012;81:430-431.

41 Vanwalleghem J, Zachee P, Kuypers D, et al: Renal transplantation for end-stage renal disease due to paroxysmal nocturnal haemoglobinuria. Nephrol Dial Transplant 1998;13: 3250-3252.

42 Hillmen P, Young NS, Schubert J, et al: The complement inhibitor eculizumab in paroxysmal nocturnal hemoglobinuria. N Engl J Med 2006;355:1233-1243.

43 Wolfe RA, Ashby VB, Milford EL, et al: Comparison of mortality in all patients on dialysis, patients on dialysis awaiting transplantation, and recipients of a first cadaveric transplant. N Engl J Med 1999:341:1725-1730.
Renal Involvement in Paroxysmal

Nocturnal Hemoglobinuria
Nephron Clin Pract 2013;123:28-35

DOI: $10.1159 / 000351345$ 